\title{
Progression of planovalgus deformity in patients with cerebral palsy
}

Jae Jung Min ${ }^{1+}$, Soon-Sun Kwon ${ }^{2 \dagger}$, Ki Hyuk Sung ${ }^{1}$, Kyoung Min Lee ${ }^{1}$, Chin Youb Chung ${ }^{1}$ and Moon Seok Park ${ }^{1 *}$ (D)

\begin{abstract}
Background: Analyzing radiographic changes of pes planovalgus(PV) deformity of cerebral palsy(CP) patients according to age and influencing factors.

Methods: CP patients with PV deformity younger than 18 years old who had undergone more than a year of follow-up with at least two standing foot radiographs were included. Anteroposterior and lateral talo-first metatarsal(talo-1stMT), talo-second metatarsal(talo-2ndMT), and hallux valgus(HV) angles were measured on the radiographs. The rate of progression was adjusted by multiple factors using the linear mixed model, with the Gross Motor Function Classification System(GMFCS) level as the fixed effect and age and each subject as random effects.

Results: Overall, 194 patients were enrolled in this study, and 1272 standing foot radiographs were evaluated. The AP talo- $2^{\text {nd }}$ MT angle progressed by $0.59^{\circ}(p<0.0001)$ and $0.64^{\circ}(p=0.0007)$ in GMFCS level II and III patients, respectively; however, there was no significant change in GMFCS level I patients $(p=0.3269)$. HV was significantly affected by age in all three GMFCS groups; it increased by $0.48^{\circ}(p<0.0001), 0.66^{\circ}(p<0.0001)$, and $1.19^{\circ}(p<$ $0.0001)$ for levels I, II, and III, respectively. The lateral talo-1stMT angle showed improvements in GMFCS level I and II patients $\left(0.43^{\circ}, p<0.0001\right.$, and $0.61^{\circ}, p<0.0001$, respectively). In GMFCS level III patients, there was no significant improvement in the lateral talo- $1^{\text {st }}$ MT angle $(p=0.0535)$.
\end{abstract}

Conclusions: The GMFCS level was the single most important factor influencing the progression of radiographic indices in PV deformity in CP. The AP talo- ${ }^{\text {st }} M T$ and talo-2ndMT angles progressed in patients with GMFCS levels II and III. Physicians should take this result into consideration when planning the timing of the surgery.

Level of evidence: Prognostic Level IV.

Keywords: Pes planovalgus, Talo-first metatarsal angle, Talo-second metatarsal angle, Progression, Cerebral palsy

\section{Background}

Pes planovalgus(PV) is one of the most common foot deformities in patients with cerebral palsy(CP), especially in patients with diplegia and quadriplegia [1]. PV in CP most likely results from muscle imbalance and spasticity in a skeletally developing foot [2]. Due to prominent talar head, PV frequently leads to pain during weight-bearing. Furthermore, in patients with CP, PV results in lever-arm dysfunction [3]: not only is PV a prototype of a flexible

\footnotetext{
*Correspondence: pmsmed@gmail.com

The study was performed at the Department of Orthopedic Surgery, Seoul National University Bundang Hospital, Gyeonggi, Korea

${ }^{\dagger}$ Jae Jung Min and Soon-Sun Kwon contributed equally to this work.

${ }^{1}$ Department of Orthopedic Surgery, Seoul National University Bundang

Hospital, 82 Gumi-ro 173 Beon-gil, Bundang-Gu, Sungnam, Gyeonggi 13620,

South Korea

Full list of author information is available at the end of the article
}

lever arm, but it also causes malrotated lever-arm dysfunction by shortening foot lever due to the externally rotated or abducted forefoot [3-6]. In patients who already have weak muscles, lever-arm dysfunctions further worsen their ambulatory function [3].

Idiopathic PV in children and adolescents improves as patients grow older [7-9], as seen in crosssectional studies $[8,9]$, and its spontaneous recovery has also been proven radiographically in a longitudinal study [7]. The anteroposterior(AP) talo-1stMT and lateral talo-1stMT angles improved annually until skeletal maturity [7]. However, PV deformity in $\mathrm{CP}$ may not follow this pattern, since underlying condition does not disappear. While spasticity continuously affects the extremities, forefoot dragging due to malrotated lever and Achilles contractures may further 
interrupt spontaneous improvement observed in idiopathic PV [5]. Also, recurrence of PV after calcaneal lengthening is greater in GMFCS III and IV patients, implying that PV can progress even after the surgical procedure [10]. However, there are few studies that invistigated progression of $\mathrm{PV}$ in $\mathrm{CP}$ patients.

Therefore, the purpose of this study was to evaluate the trend in radiologic changes of $\mathrm{CP}$ patients with PV deformity according to age and identify influencing factors.

\section{Methods}

\section{Patients and study design}

This retrospective study was approved by the institutional review board of tertiary referral center for $\mathrm{CP}$ (IRB no. B-1905-541-106). Informed consent was waived due to retrospective nature of the study.

We screened patients seen between May 2003 and May 2019 via clinical data warehouse to identify those with $\mathrm{CP}$ who were $\leq 18$ years of age, who had been followed up for more than 1 year, with at least two standing AP and lateral foot radiographs.

After screening, two authors(JJM and MSP) reviewed and screened medical records and radiographs. The exclusion criteria were (1)incorrect diagnosis, (2)varus or neutral foot at initial presentation i.e., talo- $2^{\text {nd }} \mathrm{MT}$ angle $<10^{\circ}$, (3)inadequate radiographs, such as inadequate weight-bearing, (4)involved side of hemiplegia, and (5)GMFCS levels IV and V. For those who had undergone intervention for foot deformity, such as calcaneal lengthening or talonavicular fusion, postsurgical radiographs taken on the operated side were excluded (Fig. 1). Standing AP and lateral foot radiographs were taken with a UT 2000 X-ray machine(Philips Research, Eindhoven, The Netherlands) at a source-to-image distance of approximately $100 \mathrm{~cm}$ with the patient standing barefoot. The X-ray machine settings ranged from 46 to $50 \mathrm{kVp}$ and 4.5 to $5 \mathrm{mAs}$, according to each patients' body size. All radiographic images were digitally acquired with the use of a picture archiving and communication system(PACS) (INFINITT Healthcare, Seoul, Korea), and measurements were subsequently carried out with the use of PACS software.

\section{Consensus building and measurement}

Five authors(JJM, KHS, KML, and MSP, who are orthopedic surgeons with $2,16,17$, and 19 years of experience, respectively, and S-SK, a statistician) agreed on the indices to be measured in the radiographs. Previous studies were reviewed [7, 11-15], and one of the authors(JJM) pooled seven indices relevant to the PV and hallux valgus(HV) that have been established with convergent and discriminant validities and intra, inter-rater reliabilities [12]: naviculocuboid(NC) overlap, AP talonavicular(TN) coverage angle, AP and lateral talo- $1^{\text {st }} \mathrm{MT}$ angles, AP

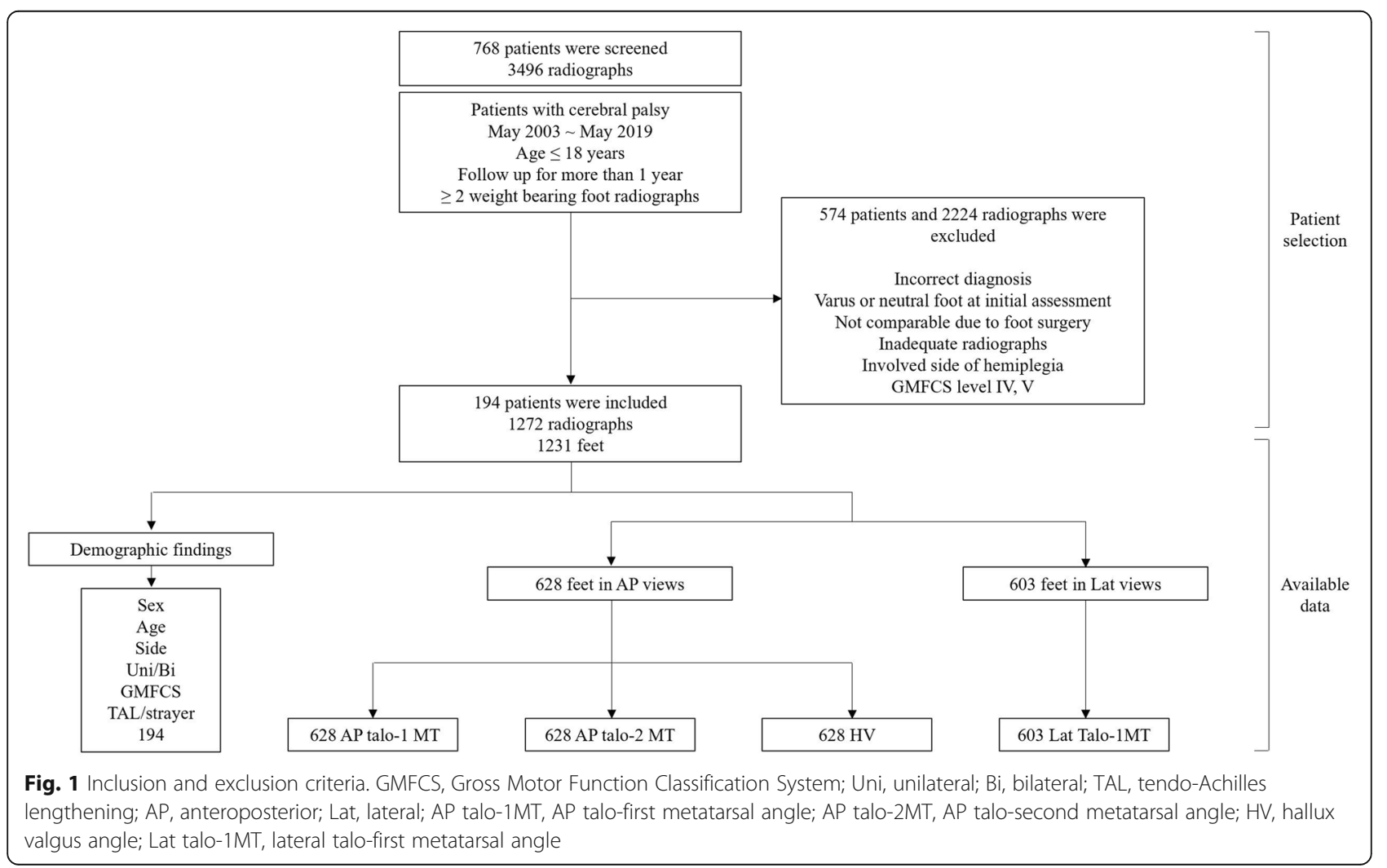




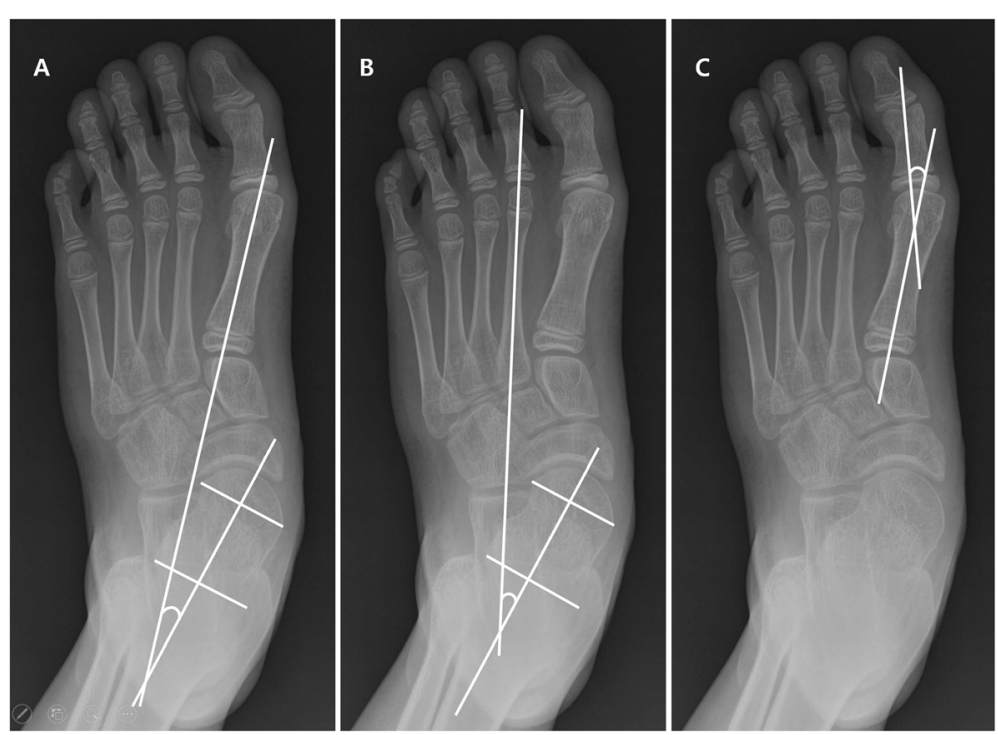

Fig. 2 a The anteroposterior talo-first metatarsal angle is the angle between the line bisecting the longitudinal axis of the first metatarsal bone and the line bisecting the longitudinal axis of the talus on standing anteroposterior plain foot radiograph. $\mathbf{b}$ The anteroposterior talo-second metatarsal angle is the angle between the line bisecting the longitudinal axis of the second metatarsal bone and the line bisecting the longitudinal axis of the talus on standing anteroposterior plain foot radiograph. c The hallux valgus angle is the angle between the longitudinal axis of the first proximal phalanx and the longitudinal axis of the first metatarsus

talo- $2^{\text {nd }} \mathrm{MT}$ angles, HV angle, and intermetatarsal angle. In our study, NC overlap and AP TN coverage angle were excluded due to incomplete ossification of the navicular bones in younger patients, making evaluation of indices that include the navicular bone difficult. We have also noted that not only does PV progress over time, but $\mathrm{HV}$ also progressed in $\mathrm{CP}$ patients, which may disturb the talo- $1^{\text {st }} \mathrm{MT}$ angle. Therefore, we introduced the talo$2^{\text {nd }} \mathrm{MT}$ angle as a main index in our evaluation.

Consequently, the $\mathrm{AP}$ and lateral talo- $1^{\text {st }} \mathrm{MT}$, AP talo- $2{ }^{\text {nd }} \mathrm{MT}$, and $\mathrm{HV}$ angles were the four indices chosen. We used AP and lateral talo- $1^{\text {st }} \mathrm{MT}$ angles and AP talo- $2{ }^{\text {nd }} \mathrm{MT}$ angle as surrogate indices for the progression of $\mathrm{PV}$ and the $\mathrm{HV}$ angle as surrogate for the progression of $\mathrm{HV}$.
The AP talo- $1^{\text {st }}$ and talo- $2^{\text {nd }}$ MT angles are the angles between a line bisecting longitudinal axis of first and second metatarsal bones, respectively, and the line bisecting the longitudinal axis of the talus on standing AP plain foot radiograph [12] (Fig. 2). The lateral talo$1^{\text {st }} \mathrm{MT}$ angle is the angle between the line bisecting the longitudinal axis of the first metatarsal bone and the line bisecting the longitudinal axis of the talus in standing lateral foot radiograph [12] (Fig. 3). The HV angle is the angle between longitudinal axis of the first proximal phalanx and first metatarsus [12, 16] (Fig. 2).

After consensus building, reliability test was conducted before primary measurements. Sample size estimation showed that a minimum of 36 feet (18 left and 18 right) radiographs should be assessed. Three authors(JJM, KHS,

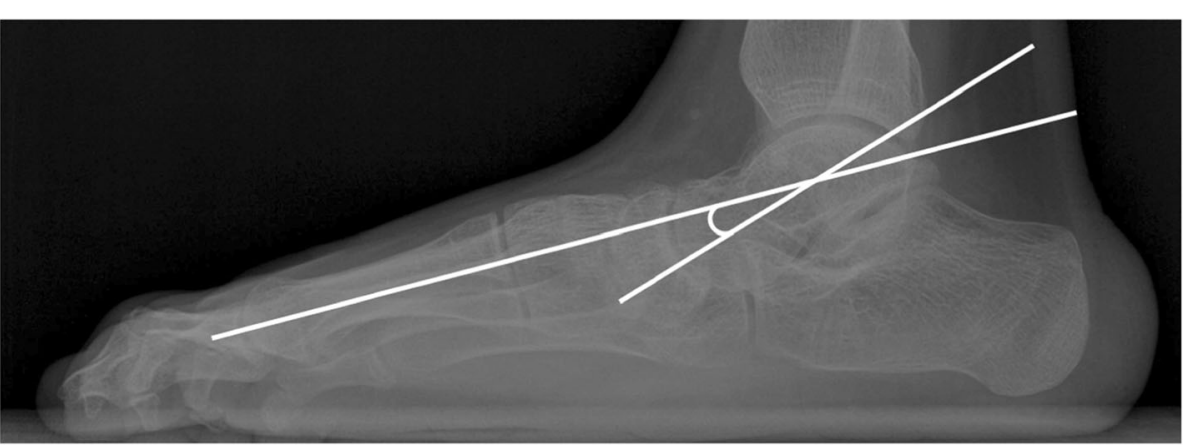

Fig. 3 The lateral talo-first metatarsal angle is the angle between the line bisecting the longitudinal axis of the first metatarsal bone and the line bisecting the longitudinal axis of the talus on the standing lateral plain foot radiograph 
and MSP) with 2, 16, and 19 years of orthopedic experience determined the interobserver reliability using intraclass correlation coefficients(ICCs), and independently measured the radiographs in a blinded fashion. Four weeks after the primary measurements, intra-observer reliability was assessed by one of the authors(JJM) who repeated the radiographic measurements.

Following reliability testing, two authors(JJM and MSP) measured indices in all the radiographs. Patient's age, sex, GMFCS level, involvement(unilateral/bilateral), history of tendo-Achilles lengthening(TAL) or gastrocnemius recession(Strayer), laterality of the feet, and the date the radiographs were also included.

\section{Building a linear mixed model}

The progression rate of the AP and lateral talo- ${ }^{\text {st }} \mathrm{MT}$, AP talo- $2^{\text {nd }} \mathrm{MT}$, and HV were adjusted by multiple factors using a linear mixed model, with sex, GMFCS, involvement (bilateral/unilateral), and TAL/Strayer as the fixed effects and laterality and each subject as random effects. The covariance structure was assumed as the variance components. The restricted maximum likelihood estimation was used to estimate parameters for the linear mixed model [17-19]. By examining the individual pattern of the annual changes in the AP and lateral talo$1^{\text {st }} \mathrm{MT}$, AP talo- $2^{\text {nd }} \mathrm{MT}$, and HV angles as well as the follow-up duration, a linear mixed model with a random slope and a random intercept was suggested. The slope was the progression rate of the AP and lateral talo$1^{\text {st }} \mathrm{MT}$, AP talo- $2^{\text {nd }} \mathrm{MT}$, and HV angles per year. Linear follow-up duration(age) effect was interpreted to evaluate the estimation of the measurements. The models were accepted as valid for estimating the responses using the Akaike information criterion(AIC) and the Bayesian information criterion(BIC). A smaller AIC or BIC value is preferred in terms of model selection [17-19].

\section{Statistical analysis}

In this study, reliability was assessed with the use of ICCs and a two-way mixed-effect model, assuming a single measurement and absolute agreement [20,21]. With the use of an ICC target value of 0.8 , a Bonett approximation was employed in setting 0.2 as the width of $95 \%$ confidence intervals(CIs). The minimal sample size was calculated to be 36 [22].

Descriptive statistics were used to summarize patient demographics and radiographic measurements. The Kolmogorov-Smirnov test was used to verify the normality of the distribution of continuous variables. Descriptive statistics included the mean, standard deviation, and frequency. A linear mixed model was used to model the progression rates, assess the covariate effect, and examine the factors that contributed significantly to the rate of progression.
All statistical analyses were performed using the SAS Statistical package version 9.4(SAS Institute, Cary, NC, USA) and $\mathrm{R}$ version 3.5.1( $\mathrm{R}$ Foundation for Statistical Computing, Vienna, Austria; ISBN 3-900,051-07-0, URL http://www.r-project.org) with the stats package 2.3. All statistics tests were two-tailed, and CIs were considered significant when they did not include zero, and $p$-values $<0.05$ were considered significant.

\section{Results}

Seven hundred sixty-eight patients met the inclusion criteria. After implementation of inclusion and exclusion criteria, 194 patients were enrolled in this study (Fig. 1). The mean age and standard deviation of the patients at the time of their first visit to the outpatient clinic was $8.6 \pm 3.1$ years, with mean follow-up of $5.2 \pm 4.0$ years. Of the 194 patients, 90 were of GMFCS I, 70 GMFCS II, and 34 were GMFCS III. 111(57\%) of 194 patients had history of TAL or Strayer at some point of their followup (Table 1).

The interobserver reliability ranged from 0.917 to 0.953, with AP talo- $1^{\text {st }} \mathrm{MT}$ angle(ICC, 0.917), lateral talo- $1^{\text {st }} \mathrm{MT}$ angle(ICC, 0.934$)$, AP talo- $2^{\text {nd }} \mathrm{MT}$ angle(ICC, 0.937), and HV angle(ICC, 0.953) (Table 2). The intraobserver reliability ranged from 0.931 to 0.961 .

In our mixed model, age was a major risk factor in GMFCS II and III patients with annual progression rate of AP talo- $2^{\text {nd }} \mathrm{MT}$ angle of $0.59^{\circ}(p<0.0001)$ and $0.64^{\circ}(p=0.0007)$ respectively, whereas age was not significant $(p=0.3269)$ in GMFCS I patients (Table 3 and Fig. 4).

The AP talo- $1^{\text {st }} \mathrm{MT}$ angle increased annually by $0.48^{\circ}$ $(p=0.0010)$ and $0.52^{\circ} \quad(p=0.0036)$ respectively in GMFCS II and III patients, whereas it was not significantly affected by age $(p=0.9819)$ in GMFCS I patients (Table 4).

HV was significantly affected by age in all three GMFCS groups, increasing by $0.48^{\circ}(p<0.0001), 0.66^{\circ}$

Table 1 Summary of patient data $(n=194)$

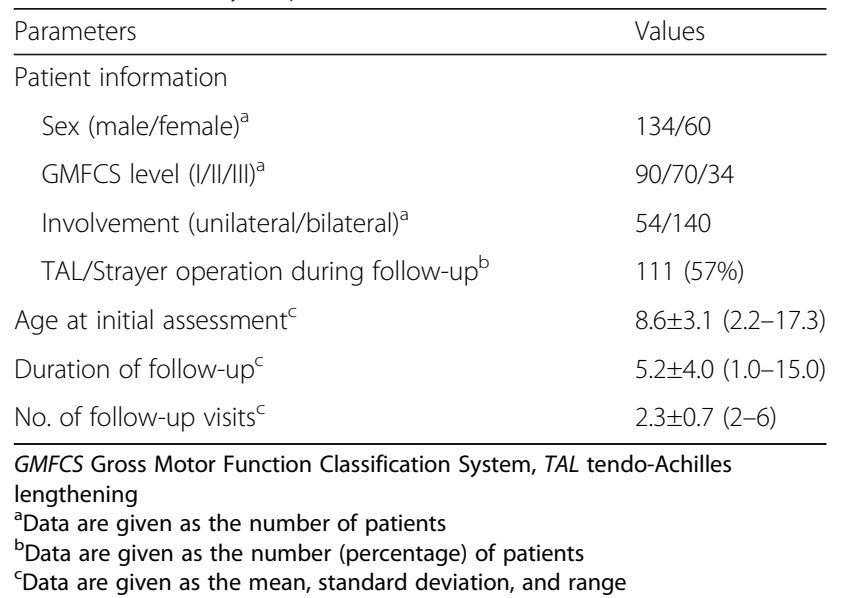


Table 2 Interobserver and intraobserver reliability of the radiographic measurements

\begin{tabular}{|c|c|c|c|c|}
\hline \multirow[b]{2}{*}{ Measurement } & \multicolumn{2}{|c|}{ Interobserver reliability } & \multicolumn{2}{|c|}{ Intraobserver reliability } \\
\hline & $\overline{I C C}$ & $95 \% \mathrm{Cl}$ & $\overline{I C C}$ & $95 \% \mathrm{Cl}$ \\
\hline AP talo-first metatarsal angle & 0.917 & $0.810-0.961$ & 0.944 & $0.893-0.971$ \\
\hline AP talo-second metatarsal angle & 0.937 & $0.888-0.966$ & 0.931 & $0.869-0.964$ \\
\hline Hallux valgus angle & 0.953 & $0.920-0.974$ & 0.961 & $0.925-0.980$ \\
\hline Lat talo-first metatarsal angle & 0.934 & $0.889-0.963$ & 0.949 & $0.903-0.974$ \\
\hline
\end{tabular}

ICC intraclass correlation coefficients, $\mathrm{Cl}$ confidence interval, $A P$ anteroposterior, Lat lateral

( $\mathrm{p}<0.0001)$, and $1.19^{\circ}(\mathrm{p}<0.0001)$ in I, II, and III patients, respectively (Table 5).

The lateral talo- $1^{\text {st }} \mathrm{MT}$ angle showed improvement with patients age. GMFCS I patients showed $0.43^{\circ}(\mathrm{p}<$ $0.0001)$ of improvement, and in level II patients showed improvement of $0.61^{\circ}(\mathrm{p}<0.0001)$. Interestingly, there was no significant improvement in the lateral talo- $1^{\text {stMT }}$ angle $(p=0.0535)$ in GMFCS III patients (Table 6).

The talo- $2^{\text {nd }}$ MT angle was $4^{\circ}$ higher in patients with GMFCS level I $(p=0.0044)$ and $3.10^{\circ}$ lower in patients with GMFCS level II $(p=0.05)$ among those who did not undergo TAL or Strayer's operation (Table 4). The

Table 3 Factors affecting the talo-second metatarsal angle according to GMFCS level

\begin{tabular}{llll}
\hline & Estimate & $95 \%$ Cl & $p$ Value \\
\hline GMFCS level I & & & \\
Intercept & 19.64 & 15.38 to 23.90 & $<0.0001$ \\
Age & 0.09 & -0.10 to 0.29 & 0.3269 \\
Sex (female) & -0.50 & -3.85 to 2.84 & 0.7644 \\
Involvement (bilateral) & 2.63 & -0.77 to 6.02 & 0.1273 \\
No TAL/Strayer & 4.00 & 1.18 to 6.83 & 0.0060 \\
Side (left) & 1.64 & -0.15 to 3.44 & 0.0724 \\
GMFCS level II & & & \\
Intercept & 24.66 & 17.60 to 31.72 & $<0.0001$ \\
Age & 0.59 & 0.31 to 0.88 & $<0.0001$ \\
Sex (female) & -0.37 & -4.19 to 3.44 & 0.8470 \\
Involvement (bilateral) & -0.95 & -7.28 to 5.38 & 0.7664 \\
No TAL/Strayer & -3.48 & -6.73 to -0.24 & 0.0357 \\
Side (left) & 0.38 & -0.94 to 2.30 & 0.4050 \\
GMFCS level III & & & \\
Intercept & 20.13 & 4.20 to 36.07 & 0.0151 \\
Age & 0.64 & 0.30 to 0.99 & $\mathbf{0 . 0 0 0 7}$ \\
Sex (female) & 4.56 & -0.89 to 10.01 & 0.0997 \\
Involvement (bilateral) & 5.26 & -10.14 to 20.66 & 0.4985 \\
No TAL/Strayer & -3.84 & -12.12 to 4.43 & 0.3580 \\
Side (left) & -0.04 & -2.37 to 2.30 & 0.9751 \\
\hline
\end{tabular}

The linear mixed model was used to estimate the factors affecting the talosecond metatarsal angle. The Akaike information criteria were 1699, 1582, and 948 for each model

GMFCS Gross Motor Function Classification System, Cl confidence interval, TAL tendo-Achilles lengthening talo- $1^{\text {st }}$ MT angle was $4.23^{\circ}$ higher in patients with GMFCS level I $(p=0.0044)$ and $3.10^{\circ}$ lower in patients with GMFCS level II $(p=0.05)$ among those who did not undergo TAL or Strayer's operation (Table 5).

\section{Discussion}

In our longitudinal assessment of progression of PV in $\mathrm{CP}$ patients, we have demonstrated that the major risk factor in PV progression is the GMFCS level. The talo$1^{\text {st }} \mathrm{MT}$ and talo- $2^{\text {nd }} \mathrm{MT}$ angles, which are surrogate indices of forefoot abduction, were significantly aggravated with age in GMFCS II and III patients $(p<0.0001, p=$ $0.0007)$. HV aggravated in all three groups $(\mathrm{p}<0.0001)$, and the lateral talo- $1^{\text {st }} \mathrm{MT}$ angle, a surrogate of planus, showed significant improvement in GMFCS I and II patients $(\mathrm{p}<0.0001, \mathrm{p}<0.0001)$, whereas there was no significant improvement in GMFCS III patients.

It has been previously noted in a longitudinal study that idiopathic PV deformity improves annually until patients reach skeletal maturity; the AP and lateral talo- $1^{\text {st }} \mathrm{MT}$ angle improved on average by $2.1^{\circ}$ and $0.7^{\circ}$ respectively annually until skeletal maturity [7] and rate of improvement decreases starting from age 10 [7]. Therefore, it had been recommended that any surgical procedure to correct the deformity, such as calcaneal lengthening [23], calcaneostop [24], and calcaneal-cuboid-cuneiform osteotomy [25], be postponed until child reaches 10 years old. In contrast, patients with $\mathrm{CP}$ forefoot abduction, as represented by the talo- $2^{\text {nd }} \mathrm{MT}$ angle, not only showed no improvement(GMFCS level I), but rather progressed by $0.59^{\circ}(p<$ $0.0001)$ and $0.64^{\circ}(p=0.0007)$ in patients with GMFCS II and III. It is therefore reasonable to believe that in patients with $\mathrm{CP}$, correction of PV should be addressed at the time of single-event multilevel surgery.

Because HV also progresses with age in patients with $\mathrm{CP}$, utilizing conventional talo- $1^{\text {st }} \mathrm{MT}$ angle to assess PV may lead to underestimation of PV progression. Hence, we used the talo- $-2{ }^{\text {nd }} \mathrm{MT}$ angle, which is more independent of the progression of HV.

The lateral talo- $1^{\text {st }} \mathrm{MT}$ angle showed improvement in patients with GMFCS I and II. This implies that planus deformity may improve over time while forefoot abduction progresses. This may be the effect of the TAL or Strayer, which improve sagittal deformity. Furthermore, 


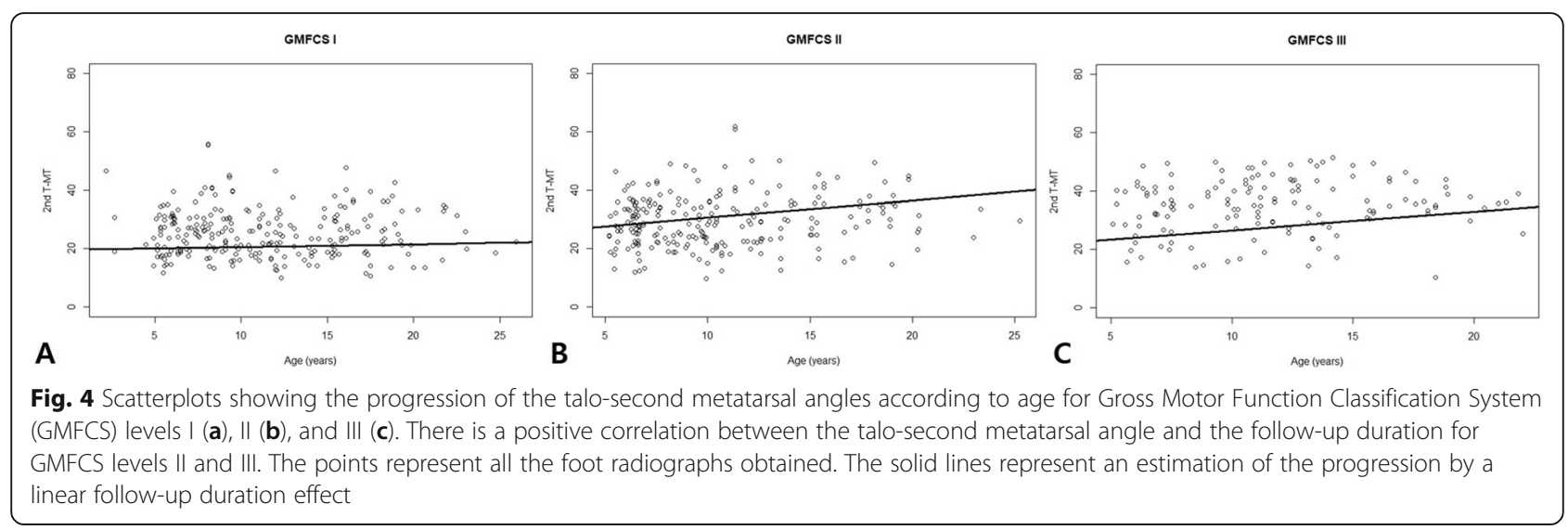

malrotated lever arm, which affects the inner ray of the foot, may have more adverse effects on the forefoot abduction than on the planus.

While discussing the clinical implications of the present study, it is crucial to address limitations. First, the study was retrospective by design. A uniform protocol was not implemented in all subjects; thus, patients

Table 4 Factors affecting the talo-first metatarsal angle according to the GMFCS level

\begin{tabular}{llll}
\hline & Estimate & $95 \%$ Cl & $p$ Value \\
\hline GMFCS level I & & & \\
Intercept & 12.16 & 7.88 to 16.44 & $<0.0001$ \\
Age & -0.00 & -0.20 to 0.19 & 0.9819 \\
Sex (male) & -1.86 & -5.09 to 1.38 & 0.2563 \\
Involvement (bilateral) & 2.80 & -0.53 to 6.12 & 0.0977 \\
No TAL/Strayer & 4.23 & 1.36 to 7.09 & 0.0044 \\
Side (left) & 1.85 & -0.00 to 3.70 & 0.0503 \\
GMFCS level II & & & \\
Intercept & 16.68 & 9.90 to 23.46 & $<0.0001$ \\
Age & 0.48 & 0.20 to 0.76 & $\mathbf{0 . 0 0 1 0}$ \\
Sex (male) & -1.62 & -5.24 to 2.01 & 0.3785 \\
Involvement (bilateral) & -0.54 & -6.64 to 5.57 & 0.8618 \\
No TAL/Strayer & -3.10 & -6.19 to -0.00 & 0.0500 \\
Side (left) & 1.18 & -0.35 to 2.72 & 0.1296 \\
GMFCS level III & & & \\
Intercept & 12.63 & -4.53 to 29.80 & 0.1430 \\
Age & 0.52 & 0.18 to 0.86 & $\mathbf{0 . 0 0 3 6}$ \\
Sex (male) & 4.89 & -1.02 to 10.79 & 0.1036 \\
Involvement (bilateral) & 4.94 & -11.77 to 21.65 & 0.5578 \\
No TAL/Strayer & -5.76 & -14.69 to 3.17 & 0.2026 \\
Side (left) & 0.31 & -1.91 to 2.54 & 0.7793 \\
\hline
\end{tabular}

Linear mixed model was used to estimate factors affecting the talo-first metatarsal angle. The Akaike information criteria were 1708, 1562, and 941 for each model

GMFCS Gross Motor Function Classification System, Cl confidence interval, TAL tendo-Achilles lengthening differed in age at initial assessment, duration of followup, and number of radiographs taken during follow-up. However, a linear mixed model consisting of fixed and random effects was selected to adjust the repeated measures or longitudinal data that are an inherent limitation of this study design. Second, confounding variables such as achilles tightness and patients' activity level could not

Table 5 Factors affecting the hallux valgus angle according to GMFCS level

\begin{tabular}{llll}
\hline & Estimate & $95 \%$ Cl & $p$ Value \\
\hline GMFCS level I & & & \\
Intercept & 6.34 & 3.38 to 9.31 & $<0.0001$ \\
Age & 0.48 & 0.34 to 0.61 & $<0.0001$ \\
Sex (female) & 2.29 & -0.02 to 4.59 & 0.0515 \\
Involvement (bilateral) & 1.92 & -0.44 to 4.27 & 0.1088 \\
No TAL/Strayer & -0.71 & -2.69 to 1.26 & 0.4758 \\
Side (left) & -0.45 & -1.70 to 0.82 & 0.4840 \\
GMFCS level II & & & \\
Intercept & 6.38 & 0.52 to 12.24 & 0.0332 \\
Age & 0.66 & 0.43 to 0.89 & $<\mathbf{0 . 0 0 0 1}$ \\
Sex (female) & 4.45 & 1.12 to 7.78 & 0.0093 \\
Involvement (bilateral) & -1.65 & -7.08 to 3.79 & 0.5490 \\
No TAL/Strayer & -0.94 & -3.36 to 1.48 & 0.4406 \\
Side (left) & 0.32 & -0.79 to 1.43 & 0.5693 \\
GMFCS level III & & & \\
Intercept & 2.03 & -10.04 to 14.10 & 0.7334 \\
Age & 1.19 & 0.80 to 1.59 & $<0.0001$ \\
Sex (female) & 1.55 & -2.38 to 5.47 & 0.4346 \\
Involvement (bilateral) & 0.48 & -11.22 to 12.18 & 0.9353 \\
No TAL/Strayer & 3.43 & -3.92 to 10.78 & 0.3558 \\
Side (left) & -0.21 & -2.23 to 1.80 & 0.8325 \\
\hline
\end{tabular}

Linear mixed model was used to estimate factors affecting the hallux valgus angle. The Akaike information criteria were 1525, 1449, and 908 for each model

GMFCS Gross Motor Function Classification System, Cl confidence interval, TAL tendo-Achilles lengthening 
Table 6 Factors affecting the lateral talo-first metatarsal angle according to the GMFCS level

\begin{tabular}{llll}
\hline & Estimate & $95 \%$ Cl & $p$ Value \\
\hline GMFCS level I & & & \\
Intercept & 18.65 & 14.23 to 23.07 & $<0.0001$ \\
Age & -0.43 & -0.62 to -0.25 & $<0.0001$ \\
Sex (female) & 0.23 & -3.60 to 4.06 & 0.9057 \\
Involvement (bilateral) & 2.42 & -1.28 to 6.12 & 0.1951 \\
No TAL/Strayer & -1.10 & -3.92 to 1.72 & 0.4371 \\
Side (left) & 1.33 & -0.50 to 3.16 & 0.1519 \\
GMFCS level II & & & \\
Intercept & 24.01 & 14.83 to 33.20 & $<0.0001$ \\
Age & -0.61 & -0.88 to -0.34 & $<0.0001$ \\
Sex (female) & -0.36 & -5.60 to 4.89 & 0.8923 \\
Involvement (bilateral) & 2.83 & -5.47 to 11.14 & 0.4997 \\
No TAL/Strayer & 3.15 & -0.81 to 7.12 & 0.1180 \\
Side (left) & 0.61 & -1.34 to 2.56 & 0.5344 \\
GMFCS level III & & & \\
Intercept & 49.81 & 29.71 to 69.90 & $<0.0001$ \\
Age & -0.50 & -1.01 to 0.01 & 0.0535 \\
Sex (female) & 1.86 & -4.74 to 8.46 & 0.5766 \\
Involvement (bilateral) & -14.52 & -34.07 to 5.03 & 0.1433 \\
No TAL/Strayer & 8.20 & -3.05 to 19.44 & 0.1506 \\
Side (left) & -1.56 & -4.43 to 1.30 & 0.2806 \\
\hline Ling mixed mode was used to & & & \\
\hline
\end{tabular}

Linear mixed model was used to estimate factors affecting the lateral talo-first metatarsal angle. The Akaike information criteria were 1563, 1576, and 1049 for each model

GMFCS Gross Motor Function Classification System, Cl confidence interval, TAL tendo-Achilles lengthening

be identified with our data. With our data, it is difficult to conclude that TAL or Strayer's operation is a risk factor for changes in the talo-metatarsal angle. These limitations may be explored in future study in order to verify effects of such variables.

\section{Conclusion}

In conclusion, the GMFCS level was the most important factor influencing the progression of radiographic indices in PV. The AP talo- $1^{\text {st }}$ and talo- $2^{\text {nd }} \mathrm{MT}$ angles progressed in patients with GMFCS II and III. Physicians should take this result into consideration when planning the timing of the surgery.

\section{Abbreviations}

AP: Anteroposterior; Cl: Confidence interval; CP: Cerebral palsy; GMFCS: Gross Motor and Functional Classification System; HV: Hallux valgus; PV: Pes planovalgus; TAL: Tendoachcilles lengthening; Talo-1st MT, talo-2nd MT angle: Talus-first metatarsal angle, talus-second metatarsal angle

\section{Acknowledgments}

The authors thank Hyun Joo Ryu and Eun Young Lee for technical support.

\section{Authors' contributions}

All authors on this manuscript (JJM, S-SK, KHS, KML, CYC and MSP) made significant contributions to the study design. JJM, S-SK, KML and CYC were involved in acquisition of data. JJM, S-SK and MSP were involved in the analysis and interpretation of data, as well as drafting the manuscript. All authors gave final approval of the version to be published.

\section{Funding}

This research was supported by the Basic Science Research Program through the National Research Foundtion of Korea (NRF) funded by the Ministry of Science and ICT (No.NRF-2017R1A2B4007412), the Ministry of SMEs and Startups (grant no. S2566948) and the SNUBH Research Fund (grant no. 132015-008). The funding body helped with data collection and analysis of this data.

\section{Availability of data and materials}

The data set supporting the conclusion of this article is available on request to the corresponding author.

\section{Ethics approval and consent to participate}

This study was approved by the institutional review board of our tertiary referral center for CP (IRB no. B-1905-541-106). Informed consent was waived due to retrospective nature of the study.

\section{Consent for publication}

Not applicable.

\section{Competing interests}

The authors declare that they have no competing interests.

\section{Author details}

${ }^{1}$ Department of Orthopedic Surgery, Seoul National University Bundang Hospital, 82 Gumi-ro 173 Beon-gil, Bundang-Gu, Sungnam, Gyeonggi 13620, South Korea. ${ }^{2}$ Department of Mathematics, College of Natural Sciences, Ajou University, Gyeonggi, South Korea.

Received: 29 November 2019 Accepted: 19 February 2020 Published online: 03 March 2020

\section{References}

1. Kedem P, Scher DM. Foot deformities in children with cerebral palsy. Curr Opin Pediatr. 2015;27(1):67-74.

2. Kadhim M, Miller F. Pes planovalgus deformity in children with cerebral palsy: review article. J Pediatr Orthop B. 2014;23(5):400-5.

3. Theologis T. Lever arm dysfunction in cerebral palsy gait. J Child Orthop. 2013;7(5):379-82.

4. Sees JP, Miller F. Overview of foot deformity management in children with cerebral palsy. J Child Orthop. 2013;7(5):373-7.

5. Kalkman BM, Bar-On L, Cenni F, Maganaris CN, Bass A, Holmes G, Desloovere K, Barton GJ, O'Brien TD. Achilles tendon moment arm length is smaller in children with cerebral palsy than in typically developing children. J Biomech. 2017:56:48-54.

6. Novacheck TF, Gage JR. Orthopedic management of spasticity in cerebral palsy. Childs Nerv Syst. 2007:23(9):1015-31.

7. Park MS, Kwon SS, Lee SY, Lee KM, Kim TG, Chung CY. Spontaneous improvement of radiographic indices for idiopathic planovalgus with age. J Bone Joint Surg Am. 2013;95(24):e193 (191-198).

8. Staheli LT, Chew DE, Corbett M. The longitudinal arch. A survey of eight hundred and eighty-two feet in normal children and adults. J Bone Joint Surg Am. 1987;69(3):426-8.

9. Pfeiffer M, Kotz R, Ledl T, Hauser G, Sluga M. Prevalence of flat foot in preschool-aged children. Pediatrics. 2006;118(2):634-9.

10. Cho BC, Lee $H$, Chung CY, Sung KH, Lee KM, Kwon SS, Moon SJ, Kim J, Lim $H$, Park MS. Undercorrection of planovalgus deformity after calcaneal lengthening in patients with cerebral palsy. J Pediatr Orthop B. 2018;27(3): 206-13.

11. Lee SY, Chung CY, Park MS, Sung KH, Ahmed S, Koo S, Kang DW, Lee KM. Radiographic measurements associated with the natural progression of the hallux Valgus during at least 2 years of follow-up. Foot Ankle Int. 2018:39(4): 463-70. 
12. Lee KM, Chung CY, Park MS, Lee SH, Cho JH, Choi IH. Reliability and validity of radiographic measurements in hindfoot varus and valgus. J Bone Joint Surg Am. 2010;92(13):2319-27.

13. Sung KH, Kwon SS, Park MS, Lee KM, Ahn J, Lee SY. Natural progression of radiographic indices in juvenile hallux valgus deformity. Foot Ankle Surg. 2019;25(3):378-82.

14. Vanderwilde R, Staheli LT, Chew DE, Malagon V. Measurements on radiographs of the foot in normal infants and children. J Bone Joint Surg Am. 1988;70(3):407-15.

15. Davids JR, Gibson TW, Pugh LI. Quantitative segmental analysis of weightbearing radiographs of the foot and ankle for children: normal alignment. J Pediatr Orthop. 2005;25(6):769-76.

16. Lee KM, Ahn S, Chung CY, Sung KH, Park MS. Reliability and relationship of radiographic measurements in hallux valgus. Clin Orthop Relat Res. 2012; 470(9):2613-21.

17. Kwon SS, Lee KM, Chung CY, Lee SY, Park MS. An Introduction to the Linear Mixed Model for Orthopaedic Research. JBJS Rev. 2014;2(12).

18. Chambers J. Statistical models in S. Boca Raton: Chapman\&Hall: CRC; 1992.

19. Wilkinson GN, Rogers CE. Symbolic description of factorial models for analysis of variance. J Appl Stat. 1973;22:392-9.

20. Lee KM, Lee J, Chung CY, Ahn S, Sung KH, Kim TW, Lee HJ, Park MS. Pitfalls and important issues in testing reliability using intraclass correlation coefficients in orthopaedic research. Clin Orthop Surg. 2012;4(2):149-55.

21. Shrout PE, Fleiss JL. Intraclass correlations: uses in assessing rater reliability. Psychol Bull. 1979;86(2):420-8.

22. Bonett DG. Sample size requirements for estimating intraclass correlations with desired precision. Stat Med. 2002;21(9):1331-5.

23. Mosca VS. Calcaneal lengthening for valgus deformity of the hindfoot. Results in children who had severe, symptomatic flatfoot and skewfoot. J Bone Joint Surg Am. 1995;77(4):500-12.

24. Richter M, Zech S. Arthrorisis with calcaneostop screw in children corrects Talo-1st metatarsal-index (TMT-index). Foot Ankle Surg. 2013;19(2):91-5.

25. Rathjen KE, Mubarak SJ. Calcaneal-cuboid-cuneiform osteotomy for the correction of valgus foot deformities in children. J Pediatr Orthop. 1998; 18(6):775-82.

\section{Publisher's Note}

Springer Nature remains neutral with regard to jurisdictional claims in published maps and institutional affiliations.

Ready to submit your research? Choose BMC and benefit from:

- fast, convenient online submission

- thorough peer review by experienced researchers in your field

- rapid publication on acceptance

- support for research data, including large and complex data types

- gold Open Access which fosters wider collaboration and increased citations

- maximum visibility for your research: over $100 \mathrm{M}$ website views per year

At $\mathrm{BMC}$, research is always in progress.

Learn more biomedcentral.com/submissions 